\title{
EXPANDED VERY LARGE ARRAY OBSERVATIONS OF THE NEBULA AROUND G79.29+0.46
}

\author{
G. Umana ${ }^{1}$, C. S. Buemi ${ }^{1}$, C. Trigilio ${ }^{1}$, P. Leto ${ }^{1}$, C. Agliozzo ${ }^{2}$, A. Ingallinera ${ }^{2}$, A. Noriega-Crespo ${ }^{3}$, and J. L. Hora ${ }^{4}$ \\ ${ }^{1}$ INAF-Osservatorio Astrofisico di Catania, Via S. Sofia 78, 95123 Catania, Italy; Grazia.Umana @ oact.inaf.it \\ ${ }^{2}$ Department of Physics and Astronomy, Universitá di Catania and INAF-Osservatorio Astrofisico di Catania, Via S. Sofia 78, 95123 Catania, Italy \\ ${ }^{3}$ Spitzer Science Center, California Institute of Technology, Mail Code 314-6, Pasadena, CA 91125, USA \\ ${ }^{4}$ Harvard-Smithsonian Center for Astrophysics, 60 Garden St. MS-65, Cambridge, MA 02138-1516, USA \\ Received 2011 April 14; accepted 2011 June 9; published 2011 August 29
}

\begin{abstract}
We have observed the radio nebula surrounding the Galactic luminous blue variable candidate G79.29+0.46 with the Expanded Very Large Array (EVLA) at $6 \mathrm{~cm}$. These new radio observations allow a morphological comparison between the radio emission, which traces the ionized gas component, and the mid-IR emission, a tracer of the dust component. The InfraRed Array Camera $(8 \mu \mathrm{m})$ and the Multiband Imaging Photometer for Spitzer $(24 \mu \mathrm{m}$ and $70 \mu \mathrm{m}$ ) images have been reprocessed and compared with the EVLA map. We confirm the presence of a second shell at $24 \mu \mathrm{m}$ and also provide evidence for its detection at $70 \mu \mathrm{m}$. The differences between the spatial morphology of the radio and mid-IR maps indicate the existence of two dust populations, the cooler one emitting mostly at longer wavelengths. Analysis of the two dusty, nested shells have provided us with an estimate of the characteristic timescales for shell ejection, providing important constraints for stellar evolutionary models. Finer details of the ionized gas distribution can be appreciated thanks to the improved quality of the new $6 \mathrm{~cm}$ image, most notably the highly structured texture of the nebula. Evidence of interaction between the nebula and the surrounding interstellar medium can be seen in the radio map, including brighter features that delineate regions where the shell structure is locally modified. In particular, the brighter filaments in the southwest region appear to frame the shocked southwestern clump reported from CO observations.
\end{abstract}

Key words: circumstellar matter - infrared: stars - stars: early-type - stars: individual (G79.29+0.46) - stars: winds, outflows

Online-only material: color figures

\section{INTRODUCTION}

Massive stars play a fundamental role in the evolution of galaxies. They are major contributors to the interstellar UV radiation and, via their strong stellar winds, provide enrichment of processed material (gas and dust) and mechanical energy to the interstellar medium. Despite their importance, the details of post-main-sequence evolution of massive stars are still poorly understood. Recent evolutionary models suggest that luminous blue variables (LBVs) and related transition objects may play a key role in the massive star evolution, representing a crucial phase during which a star loses most of its $\mathrm{H}$ envelope (Lamers et al. 2001). More recently, it has also been pointed out that LBVs might be direct supernova progenitors (Kotak \& Vink 2006; Smith et al. 2008), enhancing their importance in the framework of stellar evolution.

LBVs are luminous (intrinsically bright, $L \sim 10^{6} L_{\odot}$ ) objects, exhibiting different kinds of photometric and spectroscopic variabilities. They are massive $\left(M \sim 20-120 M_{\odot}\right)$, characterized by intense mass-loss rates $\left(10^{-6}\right.$ to $\left.10^{-4} M_{\odot} \mathrm{yr}^{-1}\right)$, which can also occur in the form of eruptive events. Although eruptive events have been witnessed very rarely (e.g., $\eta$ Car and P Cyg) the presence of extended, dusty circumstellar nebulae around LBVs (LBVNe) suggests that they are a common aspect of LBV behavior (Weis 2008). There are, however, many aspects of LBV phenomenon that are not completely understood. Among these are the total mass lost during the LBV phase (a key parameter necessary to test evolutionary models), the origin and shaping of the LBVNe, and how the mass-loss behavior (single versus multiple events, bursts) is related to the physical parameters of the central object.
The mass-loss archaeology of the central object can be recovered from an analysis of its associated nebula. A successful approach is based on a synergistic use of different techniques, at different wavelengths, that allows one to analyze the several emitting components coexisting in the nebula. In particular, a detailed comparison of mid-IR and radio maps, with comparable spatial resolution, has provided estimates of both ionized gas and dust masses and allowed us to sort out morphological differences in the maps which can be associated with mass-loss behavior during the LBV phase (Buemi et al. 2010; Umana et al. 2010).

\subsection{The Nebula Surrounding G79.29+0.46}

G79.29+0.46 is considered an LBV candidate because its observed properties to date do not meet the requirements of spectral and photometric variability to be accepted as a bona fide member. However, $H_{\alpha}$ variations, suggestive of mass-loss variability during $\mathrm{S}$-Dor variations, have recently been reported by Vink et al. (2008).

The highly symmetric ringlike structure surrounding G79.29+0.46 was first pointed out by Wendker et al. (1991). The thermal nature of the continuum radio emission was determined by Higgs et al. (1994), who suggested that the ringlike nebula is an ionized shell of swept-up interstellar material. By examining the IRAS high-resolution images, Waters et al. (1996) concluded that the ringlike nebula is a detached shell formed during an epoch of high mass loss $\left(\sim 5 \times 10^{-4} M_{\odot} \mathrm{yr}^{-1}\right)$ followed by a quieter period. This scenario is consistent with G79.29+0.46 being an LBV, where different mass loss events may have occurred in the recent past.

More recently, the Spitzer Space Telescope has provided highsensitivity, high-resolution InfraRed Array Camera (IRAC) and 
Table 1

Observational Summary

\begin{tabular}{lcccc}
\hline \hline $\begin{array}{l}\text { Observing } \\
\text { Dates }\end{array}$ & $\begin{array}{c}\text { EVLA } \\
\text { Conf. }\end{array}$ & $\begin{array}{c}\text { Frequency } \\
(\mathrm{GHz})\end{array}$ & $\begin{array}{c}\text { Integration Time } \\
(\text { minutes })\end{array}$ & Phase Cal \\
\hline 2010 Jun 1 & D & 1.4 & 90 & $\mathrm{~J} 2052+3635$ \\
2010 Jun 11 & D & 4.9 & 90 & $\mathrm{~J} 2015+3710$ \\
2010 Dec 1 & C & 1.4 & 90 & $\mathrm{~J} 2015+3710$ \\
2010 Dec 5 & C & 4.9 & 90 & $\mathrm{~J} 2015+3710$ \\
\hline
\end{tabular}

Multiband Imaging Photometer for Spitzer (MIPS) maps (The Cygnus-X Spitzer legacy program; Hora et al. 2010) which provides a better understanding of the dust properties (Kraemer et al. 2010). Moreover, the detection of CO millimeter emission in G79.29+0.46 (Rizzo et al. 2008) demonstrates that another component, consisting of molecular gas, is present in the surroundings of this star and should be included in the budget of the total mass lost from the central object. In their CO maps of G79.29+0.46, Rizzo et al. (2008) identified components of warm and dense molecular gas whose morphology closely resembles that of the extended IRAS nebula. They also reported the presence of a shock front, interpreted as a natural consequence of different wind regimes during the central object's evolution.

Despite the wealth of new mid-IR and millimeter observations of the sources, our knowledge of the radio emission has been limited to the 1988 VLA data reported by Higgs et al. (1994) and on the 1400 and $350 \mathrm{MHz}$ Westerbork observations, carried out between 1996 and 1997 and reported by Setia Gunawan et al. (2003). In this Letter, we present new Expanded Very Large Array (EVLA) observations with sufficient dynamical range, sensitivity, and angular resolution to provide a good match to the Spitzer images. The EVLA observations allow, for the first time, a detailed morphological comparison with other maps tracing the different components of the nebula.

\section{OBSERVATIONS AND DATA REDUCTION}

\subsection{EVLA Observations}

Radio observations of G79.29+0.46 and its associated nebula were carried out with the EVLA ${ }^{5}$ on 2010 June 1 and 11 and 2010 December 1 and 5. The source was observed at two frequencies (1.4 and $5 \mathrm{GHz}$ ), with a total bandpass of $256 \mathrm{MHz}$ and in two different array configurations (D and C). For each configuration and frequency, the same observing scheme was followed, namely, a 5 minute on-source scan preceded and followed by a 1 minute scan on the phase calibrator, for a total of 75 minutes on-source integration time. The source J0137+3309 was used to fix the absolute amplitude scale and to correct for the bandpass. Table 1 contains a summary of the observing details. The data reduction was carried out within the Common Astronomical Software Applications (CASA) package, version 3.0.2. At each frequency, the data from each configuration were first independently calibrated and then combined into a single UV data set. The imaging process was performed by setting the Briggs robust parameter equal to 0 , a compromise between uniform weighting of the baseline for highest angular resolution and natural weighting for highest sensitivity. We also used a multi-scale CLEANing algorithm, intended for high resolution image but sensitive to extended structures (Brogan et al. 2006),

\footnotetext{
5 The National Radio Astronomy Observatory is a facility of the Nationa Science Foundation operated under cooperative agreement by Associated Universities, Inc.
}

resulting in a single image with an rms noise of $0.07 \mathrm{mJy}^{\text {beam }}{ }^{-1}$ and a synthetic beam of 4 .' $56 \times 3$ ". 09 for the $5 \mathrm{GHz}$ observations, and an rms noise of $0.5 \mathrm{mJy}^{\text {beam }}{ }^{-1}$ and a synthetic beam of $18^{\prime \prime} .5 \times 18^{\prime \prime} .6$ for the $1.4 \mathrm{GHz}$ observations.

\subsection{Spitzer Data \\ 2.2.1. IRAC Data}

Infrared imaging of the field including G79.29+0.46 was performed at 3.6, 4.5, 5.8, and $8.0 \mu \mathrm{m}$ with IRAC (Fazio et al. 2004) on Spitzer (Werner et al. 2004). All available data for G79.29+0.46 from the cryogenic Spitzer mission archive were used, including AORIDs 6050560, 17330688, 27106560, and 27107584. The observations used the 12 and 30 s HDR modes, which obtain integrations with frame times of 0.6 and $12 \mathrm{~s}$ and 1.2 and $30 \mathrm{~s}$, respectively. The basic calibrated data $(\mathrm{BCD})$ were retrieved from the archive (pipeline version S18.18), and additional interactive processing was performed using the imclean ${ }^{6}$ tools to remove image artifacts from bright sources, including muxbleed, banding, and column pulldown effects (Hora et al. 2004). Mosaics at each wavelength were constructed using IRACproc (Schuster et al. 2006), combining the data which was taken at different spacecraft orientations and resampling to a final image pixel size of $0{ }^{\prime \prime} 6$. Outliers due to cosmic rays or instrument artifacts not previously flagged are removed in this process. The images were combined using a weighting based on the integration time of the frames. In pixels that are saturated in the longer exposures, only the shorter frames are used in the final image.

\subsubsection{MIPS Data}

The MIPS data were taken as part of the Cygnus-X Spitzer Legacy program (PID 40184, PI: J. Hora) in the fast scanning mode with a cross scan step of $148^{\prime \prime}$ to fill the $70 \mu \mathrm{m}$ array, resulting in an integration time of $15.7 \mathrm{~s} \mathrm{pixel}^{-1}$ on a single scan leg at both 24 and $70 \mu \mathrm{m}$. The $24 \mu \mathrm{m}$ data were reprocessed using the MIPSGAL (Carey et al. 2009) data processing pipeline (Mizuno et al. 2008). The $70 \mu \mathrm{m}$ data also used the MIPSGAL pipeline (R. Paladini et al. 2011, in preparation) with the exception that the nonlinearity correction at $70 \mu \mathrm{m}$ was done per pixel using the prescription defined by the SINGS Legacy team (Dale et al. 2007), based on the behavior of the MIPS $70 \mu \mathrm{m}$ calibrators.

\section{THE RADIO NEBULA ASSOCIATED WITH G79.29+0.46}

As we intend to compare the spatial distribution of the ionized gas component, traced by the radio observations, with the morphology of the dust component, traced by the midIR observations, in the following we will analyze and discuss only the $5 \mathrm{GHz}$ data set, as that image provides details with a spatial resolution comparable to that of Spitzer observations. The morphology of the radio nebula is evident in our $6 \mathrm{~cm}$ EVLA multi-configuration image, which reveals a well-defined shell-like structure whose overall shape was previously reported by Higgs et al. (1994). However, the improved quality of our image, shown in Figure 1, allows us to discern finer details of the ionized gas distribution, most notably the highly structured texture of the nebula. There is evidence for interaction between G79.29+0.46 and the surrounding interstellar medium,

\footnotetext{
6 http://irsa.ipac.caltech.edu/data/SPITZER/docs/dataanalysistools/tools/ contributed/irac/imclean/
} 


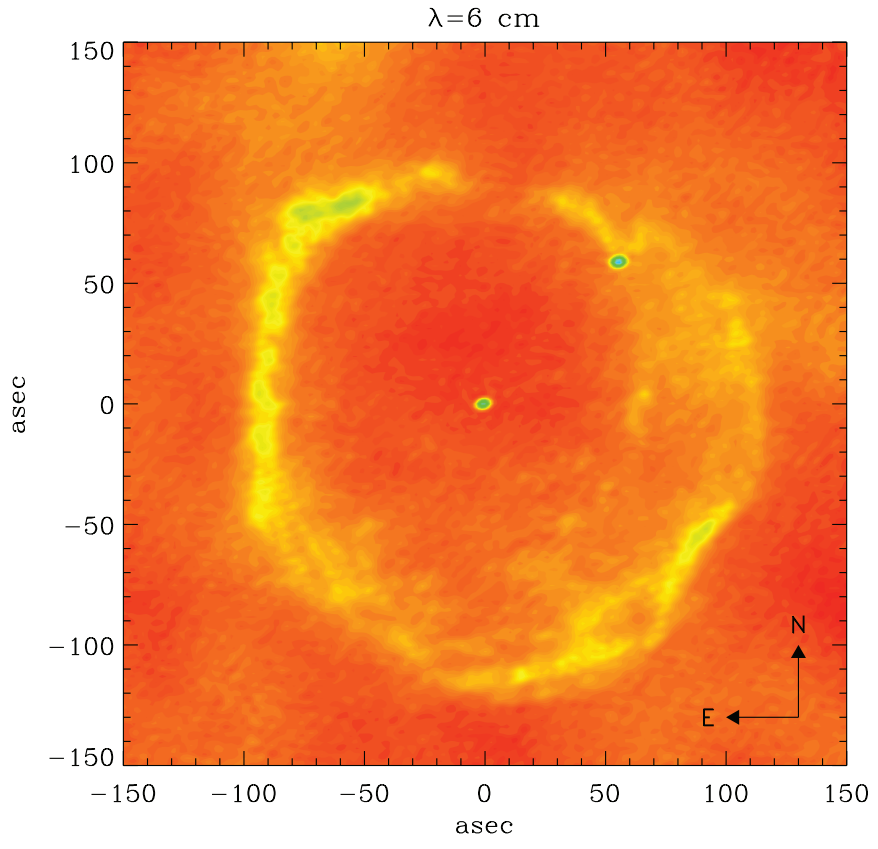

Figure 1. $6 \mathrm{~cm}$ EVLA map of G79.29+0.46 obtained by combining the data from two different array configurations, $\mathrm{C}$ and $\mathrm{D}$. The field of view is $2.5 \times 2.5$ centered on the LBV position.

(A color version of this figure is available in the online journal.)

i.e., the bright frontal structures in the northeast and the southwest regions of the nebula that delineate regions where the shell structure is locally modified. In particular, the brighter filaments in the southwest region appear to frame the shocked southwestern clump observed in CO (Rizzo et al. 2008; JimenezEsteban et al. 2010).

The central object is well detected, as is the (probably extragalactic) background object to the northwest. The position, flux density, and angular sizes of these components have been derived by fitting two-dimensional Gaussian brightness distributions to the map. We obtained a flux density of $1.51 \pm$ 0.08 and $1.88 \pm 0.09 \mathrm{mJy}$ for the central and background components, respectively. The uncertainty associated with the flux density estimation is given by

$$
\sigma=\sqrt{\left(\mathrm{RMS}_{\mathrm{tot}}\right)^{2}+\left(\sigma_{\mathrm{cal}} S_{v}\right)^{2}}
$$

where $\mathrm{RMS}_{\text {tot }}$ is the rms noise in the map and $\sigma_{\text {cal }}$ is the systematic error due to the flux calibrator (typically on the order of $3 \%$ ).

Within the errors, the derived flux densities are in agreement with those determined by Higgs et al. (1994), who derived a spectral index of $1.39 \pm 0.14$ between 5 and $8.4 \mathrm{GHz}$. They pointed out that, even though this spectral index is steeper than the canonical 0.6 , it is still consistent with mass loss from a stellar object.

Assuming that the central radio source is related to the stellar wind from the LBV, we can derive its current-day mass-loss from the standard formula (Panagia \& Felli 1975):

$$
\dot{M}=6.7 \times 10^{-4} v_{\infty} F_{v}^{3 / 4} D_{\mathrm{kpc}}^{3 / 2}\left(v \times g_{\mathrm{ff}}\right)^{-0.5} M_{\odot} \mathrm{yr}^{-1},
$$

where full ionization and cosmic abundances have been assumed, $F_{v}$ is the observed radio flux density, in mJy, and $v_{\infty}$ is the terminal velocity of the wind in $\mathrm{km} \mathrm{s}^{-1}$. The free-free Gaunt factor $g_{\mathrm{ff}}$ is approximated by $g_{\mathrm{ff}}=9.77\left(1+0.13 \log \frac{T^{3 / 2}}{v}\right)$
(Leitherer \& Robert 1991). From the radio flux density observed at $5 \mathrm{GHz}$, assuming as stellar wind velocity a value of $v \sim 110 \mathrm{~km} \mathrm{~s}^{-1}$ (Voors et al. 2000), a wind temperature of $10^{4} \mathrm{~K}$, and a distance of $1.7 \mathrm{kpc}$ (Jimenez-Esteban et al. 2010), we derive a mass loss rate of $\dot{M}=5 \times 10^{-7} M_{\odot} \mathrm{yr}^{-1}$. Vink et al. (2008) pointed out the possibility that G79.29+0.46 is associated with the nearby DR 15 region rather than with Cyg OB2. If this is the case, G79.29+0.46 is likely to be closer, at about $1 \mathrm{kpc}$. Since mass-loss scales with the distance as $D^{3 / 2}$, this would imply a reduced mass loss of a factor 0.45 . These values are smaller, but still consistent, with previous evaluations obtained by other authors using different techniques (e.g., Waters et al. 1996), but it is at least an order of magnitude smaller than the current-day mass-loss rates derived from radio measurements for other LBVs (Umana et al. 2010).

\section{IONIZED COMPONENT VERSUS DUST COMPONENTS}

The $8 \mu \mathrm{m}$ IRAC and 24 and $70 \mu \mathrm{m}$ MIPS images of G79.29+0.46 are shown in Figure 2(a). Previous versions of these images were presented by Kraemer et al. (2010) and Jimenez-Esteban et al. (2010), who both pointed out the presence of a second larger radius shell in the $24 \mu \mathrm{m}$ image. Our reprocessed maps confirm the existence of the second $24 \mu \mathrm{m}$ shell and, thanks to our improved reduction, also provide a hint of its presence at $70 \mu \mathrm{m}$. Both the 24 and $70 \mu \mathrm{m}$ maps show the same overall distribution of the dust, consistent with at least two nested dusty shells surrounding the LBV. On the contrary, in the $8 \mu \mathrm{m}$ map where a major contribution from warm dust is expected, the dust appears to be more concentrated in two southwest and southeast regions. The new EVLA radio observations allow, for the first time, a morphological comparison between the ionized gas and the dust. In Figure 2(b), the same maps are shown but now with the $6 \mathrm{~cm}$ EVLA map superimposed using white contours. Once again there is a difference between the IRAC and MIPS maps: while the nebular emission at both $24 \mu \mathrm{m}$ and $70 \mu \mathrm{m}$ is more extended than the ionized gas (radio nebula), the $8 \mu \mathrm{m}$ emission appears well contained within the ionized part of the nebula. Moreover, while the radio emission from the nebula is incomplete in the north region, the emission at $24 \mu \mathrm{m}$ and $70 \mu \mathrm{m}$ has a more uniform distribution. The apparent lack of dust in the southern regions in both $8 \mu \mathrm{m}$ and $24 \mu \mathrm{m}$ are perhaps due to an absorption effect caused by the infrared dark cloud (IRDC) which may be located in front of the southern region of the nebula.

To better visualize the spatial distribution of ionized gas relative to the dust, surface brightness profiles have been extracted along cuts through the nebula from the $6 \mathrm{~cm}, 24 \mu \mathrm{m}$, and $70 \mu \mathrm{m}$ maps. However, a direct comparison can be performed only between the $6 \mathrm{~cm}$ and the $24 \mu \mathrm{m}$ maps, as they share a comparable angular resolution, $\sim 5^{\prime \prime}$ for the $6 \mathrm{~cm}$ EVLA versus $\sim 6^{\prime \prime}$ for the $24 \mu \mathrm{m}$ MIPS map. A superposition of the $6 \mathrm{~cm}$ EVLA map on the $24 \mu \mathrm{m}$ map is shown in Figure 3. We extracted 18 cuts from each map and determined an azimuthally averaged source profile, shown in (Figure 4). The radio shell has a smaller thickness and a sharp decreasing trend in the outer part of the nebula. The dust nebula is more extended and shows a smoother distribution in the outer regions. The second shell is clearly evident in the $24 \mu \mathrm{m}$ profile, at a distance of about $\pm 200^{\prime \prime}$ from the center. At the same distance, less defined peaks are visible in the $70 \mu \mathrm{m}$ profile, smoothed out by the coarser spatial resolution. 

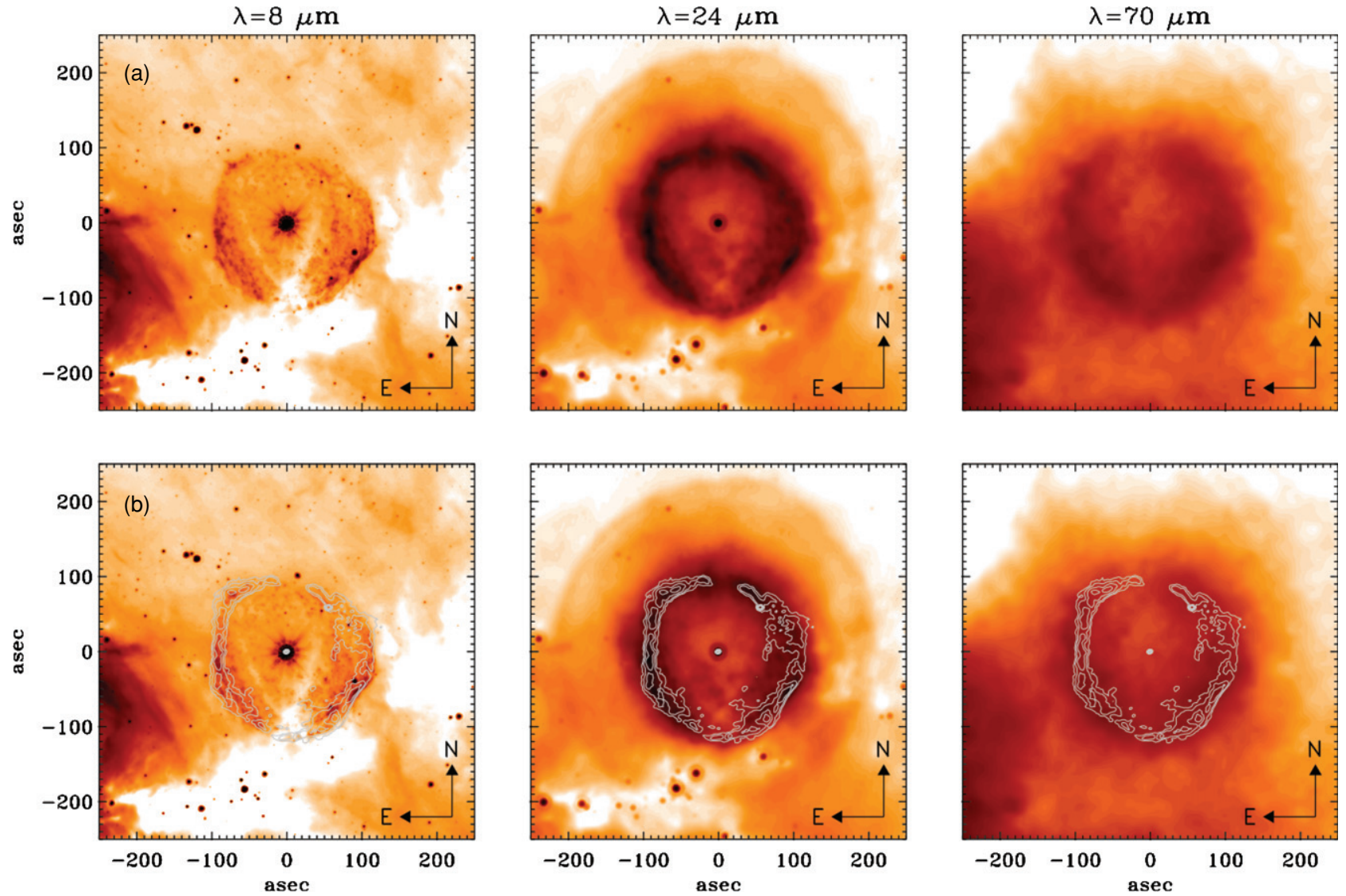

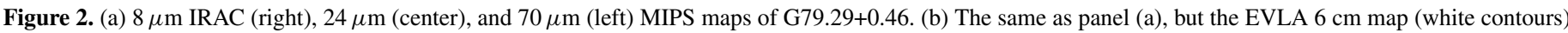

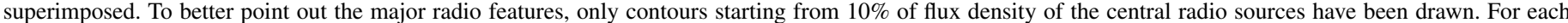
map, the field of view is $4{ }^{\prime} .2 \times 44^{\prime} .2$ centered on the LBV position.

(A color version of this figure is available in the online journal.)

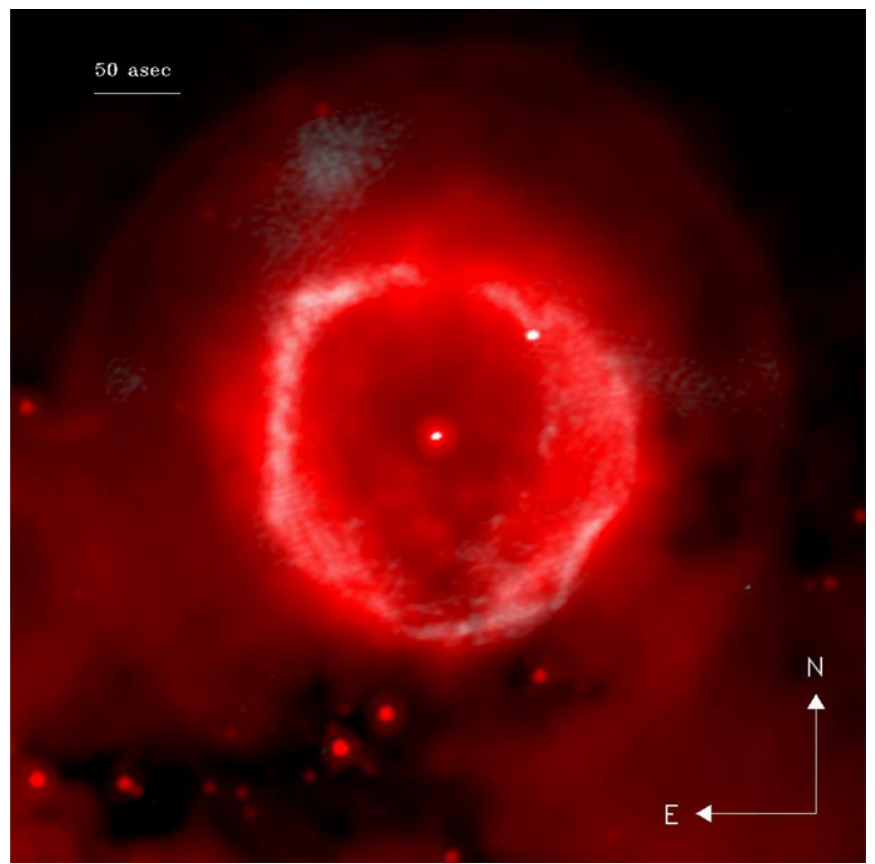

Figure 3. $6 \mathrm{~cm}$ EVLA map of G79.29+0.46 (gray) superimposed to the MIPS $24 \mu \mathrm{m}$ map (red). The field of view is $3.5 \times 3.5$ centered on the LBV position. (A color version of this figure is available in the online journal.)

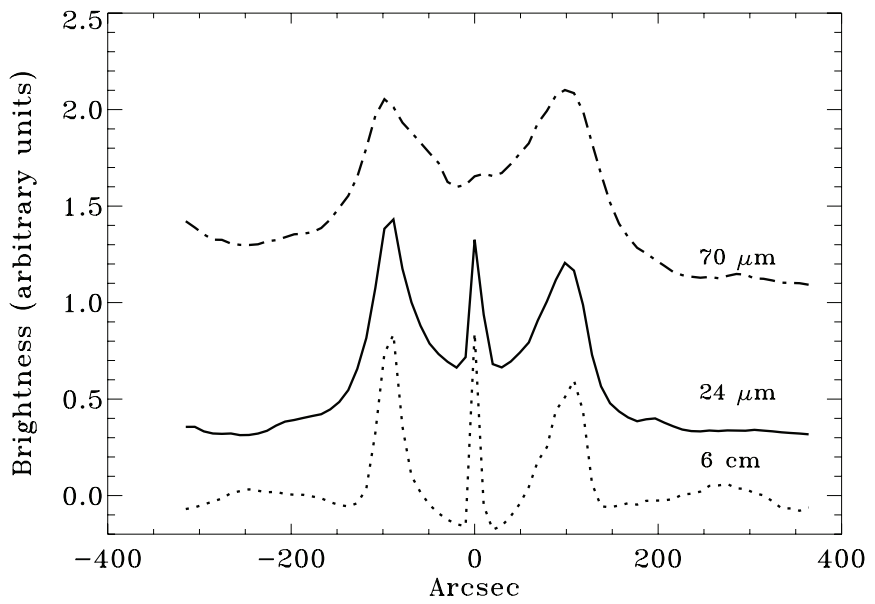

Figure 4. Averaged profiles through the nebula at $6 \mathrm{~cm}$ (dotted line), $24 \mu \mathrm{m}$ (continuous line), and $70 \mu \mathrm{m}$ (dotted slash line). Each profile has been obtained from 18 individual cuts in the corresponding map and has been shifted vertically by an arbitrary quantity for easier comparison.

\section{DISCUSSION AND CONCLUSIONS}

In this Letter, we present new radio EVLA observations of the nebula surrounding the LBV G79.29+0.46. In particular, we have analyzed the $6 \mathrm{~cm}$ map and, for the first time, compared the radio free-free, which traces the spatial distribution of the 
ionized gas, with maps recently obtained in the mid-IR, which traces the spatial distribution of the dust component.

The dust distribution observed at $8 \mu \mathrm{m}$ appears different than the distribution at 24 and $70 \mu \mathrm{m}$. The warmer dust, traced by the $8 \mu \mathrm{m}$ emission, is more concentrated in the southwest and southeast regions. Moreover, when compared with the radio nebula, the 24 and $70 \mu \mathrm{m}$ emission appears more extended, while the $8 \mu \mathrm{m}$ emission is well contained inside the radio nebula. This is consistent with the existence of two dust components: a cooler component traced by the 24 and $70 \mu \mathrm{m}$ emission and a warmer dust component, probably consisting of smaller grains, traced by the $8 \mu \mathrm{m}$ emission. This warmer component, with morphological properties quite different from those observed at longer wavelengths, could be related to the polycyclic aromatic hydrocarbon emission that has been claimed by Jimenez-Esteban et al. (2010).

An improved MIPS $70 \mu \mathrm{m}$ map, constructed using the most updated pipeline from MIPSGAL team, detects the presence of a second shell very similar to that seen at $24 \mu \mathrm{m}$. Nebular lines and dust continuum are the main possible contributors to the $24 \mu \mathrm{m}$ emission from objects embedded in a dusty nebula with a hot central component. The possibility of such a combination, with one kind of emission being predominant, has been suggested by Mizuno et al. (2010) to explain the $24 \mu \mathrm{m}$ emission detected in the MIPSGAL Bubbles. In the case of G79.29+0.46, the presence of similar shells at $24 \mu \mathrm{m}$ and at $70 \mu \mathrm{m}$, together with the lack of any prominent emission line falling within the response curve of the MIPS $24 \mu \mathrm{m}$ band, as evident from the low-resolution Infrared Spectrograph spectrum of the shell (Jimenez-Esteban et al. 2010), leaves very little doubt that the shell emission is entirely due to thermal dust emission.

The fact that there are at least two nested shells provides strong constraints on the origin of the nebula, as it is difficult to explain the presence of multiple shells in the hypothesis of a nebula consisting of swept-up interstellar medium material, as suggested by Higgs et al. (1994). It is evident that the dusty shells consist of material ejected by the central object in different mass-loss episodes.

From the $24 \mu \mathrm{m}$ emission profile, we have determined that the inner shell peaks at $100^{\prime \prime}$ and the second one at $200^{\prime \prime}$ from the central object. Such dust emission peaks can be related to the epoch when enhanced mass loss took place. Assuming a distance of $1.7 \mathrm{kpc}$ (Jimenez-Esteban et al. 2010), this corresponds to linear distances of $0.82 \mathrm{pc}$ and $1.64 \mathrm{pc}$, respectively. This implies, assuming a shell expansion velocity of $\sim 30 \mathrm{~km} \mathrm{~s}^{-1}$ (Waters et al. 1996), that the two mass-loss episodes occurred $2.7 \times 10^{4}$ and $5.4 \times 10^{4}$ years ago, or $1.6 \times 10^{4}$ and $3.1 \times 10^{4}$ years ago, if a distance of $1 \mathrm{kpc}$, as suggested by Vink et al. (2008), is used. Our results point out that mass loss can occur in different episodes, for which we derived characteristic timescales, and pose quite strong constraints that stellar evolution models must take into account.

The direct comparison of the $6 \mathrm{~cm}$ and $24 \mu \mathrm{m}$ maps (that share comparable spatial resolution) indicates that only part of the inner, brighter nebula is ionized (i.e., the nebula is ionizationbounded). The radio nebula shows a sharper profile, depicting regions where probable interaction between the second and the first dusty shells is taking place. This is evident in several regions, most notably in the northeast and in the southwest part of the nebula, where the overall quite regular shell-like morphology is locally disturbed. The mid-IR dust emission linearly depends on density and as a modified blackbody on temperature; the mid-IR emission is therefore most sensitive to dust temperature. On the other hand, assuming a uniform temperature, the thermal radio emission depends on the square of the ionized gas density. This different dependence on density means that the radio emission will emphasize features that have the largest density. This is consistent with the spatial coincidence of the bright radio features with the $\mathrm{CO}$ emission reported by Rizzo et al. (2008) and Jimenez-Esteban et al. (2010). In particular, the high critical density of the $J=3-2$ CO line $\left(n_{3} \sim 10^{4} \mathrm{~cm}^{-3}\right)$ indicates that the $\mathrm{CO}$ emission traces higher density regions, confirming that the brighter radio features delineate interactions between the shells where shocks can occur.

This work is based in part on observations made with the Spitzer Space Telescope, which is operated by the Jet Propulsion Laboratory, California Institute of Technology under a contract with NASA. Support for this work was provided by NASA through an award issued by JPL/Caltech.

\section{REFERENCES}

Brogan, C. L., Gelfand, J. D., Gaensler, B. M., Kassim, N. E., \& Lazio, T. J. W. 2006, ApJ, 639, 25

Buemi, C. S., Umana, G., Trigilio, C., Leto, P., \& Hora, J. L. 2010, ApJ, 721, 1404

Carey, S. J., Noriega-Crespo, A., Mizuno, D. R., et al. 2009, PASP, 121, 76

Dale, D. A., Gil de Paz, A., Gordon, K. D., et al. 2007, ApJ, 655, 863

Fazio, G. G., Hora, J. L., Allen, L. E., et al. 2004, ApJS, 154, 10

Higgs, L. A., Wendker, H. J., \& Landecker, T. L. 1994, A\&A, 291, 295

Hora, J. L., Fazio, G. G., Allen, L. E., et al. 2004, Proc. SPIE, 5487, 77

Hora, J. L., et al. 2010, in ASP Conf. Ser., Reionization to Exoplanets: Spitzer's Growing Legacy, in press

Jimenez-Esteban, F. M., Rizzo, J. R., \& Palau, A. 2010, ApJ, 713, 429

Kotak, R., \& Vink, J. S. 2006, A\&A, 460, L5

Kraemer, K. E., Hora, J. L., Egan, M. P., et al. 2010, AJ, 139, 2329

Lamers, H. J. G. L. M., Nota, A., Panagia, N., Smith, L. J., \& Langer, N. 2001, ApJ, 551, 754

Leitherer, C., \& Robert, C. 1991, ApJ, 377, 629

Mizuno, D. R., Carey, S. J., Noriega-Crespo, A., et al. 2008, PASP, 120, 1028

Mizuno, D. R., Kraemer, K. E., Flagey, N., et al. 2010, AJ, 139, 1542

Panagia, N., \& Felli, M. 1975, A\&A, 39, 1

Rizzo, J. R., Jimenez-Esteban, F. M., \& Ortiz, E. 2008, ApJ, 681, 355

Schuster, M. T., Marengo, M., \& Patten, B. M. 2006, Proc. SPIE, 6270, 627020

Setia Gunawan, D. Y. A., de Bruyn, A. G., Van Der Hucht, K. A., \& Williams, P. M. 2003, ApJS, 149, 123

Smith, N., Chornock, R., Li, W., et al. 2008, ApJ, 686, 467

Umana, G., Buemi, C. S., Trigilio, C., Leto, P., \& Hora, J. L. 2010, ApJ, 718, 1036

Vink, J. S., Drew, J. E., Steeghs, D., et al. 2008, MNRAS, 387, 308

Voors, R. H. M., Geballe, T. R., Waters, L. B. F. M., Najarro, F., \& Lamers, H. J. G. L. M. 2000, A\&A, 362, 236

Waters, L. B. F. M., Izumiura, H., Zaal, P. A., et al. 1996, A\&A, 313, 866

Weis, K. 2008, in Proc. International Workshop on Clumping in Hot-star Winds, ed. W. R. Hamann, A. Feldmeier, \& L. M. Oskinova (Potsdam: Univ.-Verl.) 183

Wendker, H. J., Higgs, L. A., \& Landecker, T. L. 1991, A\&A, 241, 551

Werner, M. W., Roellig, T. L., Low, F. J., et al. 2004, ApJS, 154, 1 